\title{
Ethanol tolerance and variation at the alcohol dehydrogenase (Adh) locus of Drosophila mauritiana
}

\author{
A.-Z. K. MUHAMMAD-ALI \& B. BURNET* \\ Department of Molecular Biology and Biotechnology, University of Sheffield, Sheffield S10 2UH, U.K.
}

\begin{abstract}
Drosophila mauritiana larvae are tolerant to ethanol as a supplementary nutritional resource at low concentrations. Higher levels of ethanol ( 5 per cent) in the food adversely affect larval behaviour causing reduced rates of locomotion and feeding which are associated with low larva-to-adult survival. Two codominant autosomal alleles $A d h^{\mathrm{s}}$ and $A d h^{\mathrm{F}}$ controlling variant electromorphs of alcohol dehydrogenase are indistinguishable in their effects on ethanol tolerance in this species. A comparison of larval behaviours shows that $D$. mauritiana has a lower ethanol tolerance than either of its sibling species D. melanogaster and D. simulans.
\end{abstract}

Keywords: alcohol dehydrogenase, Drosophila mauritiana, ethanol tolerance, larval behaviour, kinesis.

\section{Introduction}

Populations of Drosophila melanogaster from higher latitudes are more alcohol tolerant than equatorial populations (David \& Bocquet, 1975), and geographical variation in ethanol preference, as well as survival ability, is associated with polymorphism at the alcohol dehydrogenase (Adh) locus (David et al., 1986). The alcohol tolerance for survival of larvae and adults is positively related (van Herrewege \& David, 1985), and adults and larvae of $D$. melanogaster are more tolerant to ethanol than comparable stages in the related cosmopolitan species D. simulans (McKenzie \& Parsons, 1972; David \& Bocquet, 1976; van Herrewege \& David, 1985).

Although much of the published information on alcohol tolerance in these two cosmopolitan species has focused on the adult stage, it is likely that adaptation to environmental alcohol may be a primary target of natural selection in the larval stages also. Adaptation at the physiological and biochemical level involves ability to metabolize and detoxify ethanol present in the food. Behavioural adaptation may occur if larvae respond to alcohol in the environment by moving away from areas of high concentration, or reduce their ingestion of alcohol by adjusting their feeding rate.

The endemic island species D. mauritiana is closely related to D. simulans (Ashburner et al., 1984), but

*Correspondence. relatively little is known about its ecology. Gelfand \& McDonald (1983) have shown that the expressed levels of alcohol dehydrogenase (ADH) activity in $D$. mauritiana larvae are similar to those in $D$. simulans. Both have lower levels of ADH activity than $D$. melanogaster larvae. In preference tests, ethanol is less attractive to $D$. mauritiana at low concentrations than to $D$. simulans. On the basis of taxonomic affinity, $D$. mauritiana might be expected to be ethanol-sensitive like $D$. simulans. Here we test this prediction, bearing in mind the possibility that it may be upset by differences in their ecology and past evolutionary history.

David et al. (1980) compared the electrophoretic mobility of ADH from seven of the eight species of the Drosophila melanogaster species subgroup, excluding $D$. sechellia. They found that six species, other than $D$. melanogaster, were monomorphic for this enzyme. Presently available stocks of $D$. sechellia have also proved to be monomorphic (Burnet, unpublished results). In 1986 a massbred laboratory stock of $D$. mauritiana, originating from a wild isolate, was found to be polymorphic for different electromorphs of ADH and so provided the first observation of alcohol dehydrogenase variation in the simulans clade. This makes it possible to examine the relationship between ethanol tolerance and molecular variation at the $A d h$ locus in a member of the species subgroup other than D. melanogaster.

Larval foraging behaviour comprises a number of discrete components, the frequencies of which vary in relation to environmental factors (Green et al., 1983). 
Behavioural responses can provide a sensitive indicator of ethanol tolerance supplementary to the more widely used estimate of survival rate. Changes in the rate of feeding (phagokinesis) and locomotion (orthokinesis) are used here as behavioural measures of the effects of ethanol on D. mauritiana larvae. Drosophila mauritiana is restricted to the volcanic island of Mauritius, to the east of Madagascar, in which it is widespread and the only member of the melanogaster species subgroup (Lachaise et al., 1988). For comparison of components of larval foraging behaviour with $D$. mauritiana, we used stocks of $D$. melanogaster and $D$. simulans derived from flies caught in the wild at two circumequatorial locations on the continent of Africa.

\section{Materials and methods}

\section{Strains}

The stocks of $D$. mauritiana derived from wild isolates and maintained in the laboratory in mass culture were: Chaland, Rivilie Noire, G17, G71 and G93. These, together with stocks of $D$. erecta, $D$. orena, $D$. sechellia, $D$. teissieri and D. yakuba, were obtained from the Centre National de la Récherche Scientifique, Laboratoire de Biologie et Génétique Évolutives, Gif-surYvette, France.

Substrains of $D$. mauritiana strain G93 homozygous for $A d h^{\mathrm{S}}$ or $A d h^{\mathrm{F}}$ have been deposited at the Drosophila Stock Centre, Department of Genetics, University of Umea, Sweden.

Drosophila melanogaster were maintained in a population cage founded from a large number of females captured in a transitional habitat at Freetown, Sierra Leone. This population was monomorphic for ADH-S. Drosophila simulans were maintained as a large massbred population derived from flies captured at Nairobi, Kenya, and was similarly monomorphic for a single electromorph.

Laboratory inbred strains of $D$. melanogaster were: (i) $y$; $A d h^{\mathrm{S}} b w$; st; $e y$, (ii) $A d h^{\mathrm{F}}$, (iii) $\operatorname{lm} A d h^{\mathrm{UF}} p r c n$, and (iv) $b A d h^{\mathrm{n} 2}$ osp prcn.

\section{Electrophoresis}

Separation of alcohol dehydrogenase was performed using starch gel and a Poulik buffer system, as described by Smith (1968). A single larva, or adult fly, was gently macerated on a $6 \times 4 \mathrm{~mm}$ rectangle of Whatman $3 \mathrm{MM}$ on a chilled glass block, and one drop of Poulik gel buffer was added to the paper before insertion in the gel slot. Electrophoresis was carried out with stabilized constant voltage at $200 \mathrm{~V}$, and 25
$\mathrm{mA}$ initially, for $14-16 \mathrm{~h}$ at $4^{\circ} \mathrm{C}$. In addition to the staining reagents the incubation buffer contained ethanol and propan-2-ol, each at 0.2 per cent in $0.1 \mathrm{M}$ Tris at $\mathrm{pH} 8.6$.

\section{Larval behaviour}

Age-synchronized third instar larvae $48-50 \mathrm{~h}$ old were observed singly in a $9 \mathrm{~cm}$ glass Petri dish containing: (i) a $5 \mathrm{~mm}$ deep water-agar, overlaid with a suspension of fresh baker's yeast at 48 per cent w/v; (ii) agar gel containing 5 per cent ethanol, and overlaid with yeast suspension at 48 per cent $\mathrm{w} / \mathrm{v}$ in 5 per cent ethanol. Larvae were observed under a stereomicroscope. Locomotor activity was measured by counting the number of segmental contractions which passed anteriorily along the body of the larva during an interval of $1 \mathrm{~min}$. Feeding activity was measured by counting the number of scraping movements of the mouth hooks, actuated by the cephalopharyngeal sclerites, during an interval of $1 \mathrm{~min}$. Locomotion and feeding were recorded separately in alternate $1 \mathrm{~min}$ intervals. Twenty-five larvae from each species or genotype group were recorded first in the control yeast suspension for $30 \mathrm{~min}$, and then in yeast suspension containing 5 per cent ethanol for $30 \mathrm{~min}$. All behavioural observations were made at $25^{\circ} \mathrm{C}$.

\section{Survival}

In order to exclude any influences arising from possible induced alcohol dehydrogenase activity resulting from exposure to ethanol during embryonic development, or at eclosion, the egg collections were made using assemblies with agar containing no ethanol or acetic acid. Newly hatched first instar larvae were inoculated on to live yeast suspended in ethanol at a range of concentrations from zero up to 10 per cent. In order to maintain a constant ad libitum food supply, and a constant level of ethanol, fresh suspension was added daily until pupariation was complete. Mean survival rate was expressed as a proportion (per cent) of the initial number of first instar larvae tested. Ten replicate cultures each containing 50 larvae were used for each strain at each ethanol concentration.

\section{Results}

\section{Alcohol dehydrogenase}

Zymograms using many individuals from four strains of D. mauritiana (Chaland, Rivilie Noire, G17 and G71) proved to be monomorphic for an anodally migrating main band and lighter-staining satellite band. 
Strain G93 contained a mixture of individuals representing three discrete groups of electromorphs: (i) corresponding to that of the monomorphic strains; (ii) with a main band and a cathodally migrating satellite band; (iii) with five bands. These are illustrated in Fig. 1(b). They were designated ADH-S, ADH-F and ADH-FS, respectively. Single-pair matings of virgin flies from strain G93 were set up, and parents sacrificed after larvae appeared, to ascertain their $\mathrm{ADH}$ type. From these two pure-breeding substrains were established, one monomorphic for ADH-S, the other monomorphic for ADH-F. An analysis of the reciprocal cross, backcross and $F_{2}$ progenies showed that the different electromorphs for ADH were specified by two codominant autosomal alleles which we designated $A d h^{\mathrm{S}}$ and $A d h^{\mathrm{F}}$, respectively.

Figure 1(a) compares the ADH from all eight species of the melanogaster species subgroup in a single gel, including homozygotes for four variant alleles of $D$. melanogaster, and the two from $D$. mauritiana. Three species have electromorphs which are not unambiguously distinguishable using Poulik buffer system (simulans, sechellia and mauritiana ADH-S). The ADH of $D$. mauritiana individuals homozygous for $A d h^{\mathrm{F}}$ is very similar in electrophoretic mobility to that of D. orena.

\section{Locomotor and feeding activity of D. mauritiana larvae}

Figure 2(b) shows locomotion of early third instar larvae measured over a 30 min observation period. In yeast suspension the larvae showed a constant rate of locomotion at approximately 24 movements per min. Larvae homozygous for alleles $A d h^{\mathrm{F}}$ and $A d h^{\mathrm{S}}$ had the same rates of locomotor activity within the limits of statistical sampling error. On transfer to yeast, which was suspended in 5 per cent ethanol, there was a significant decline in locomotor activity in both genotype groups.

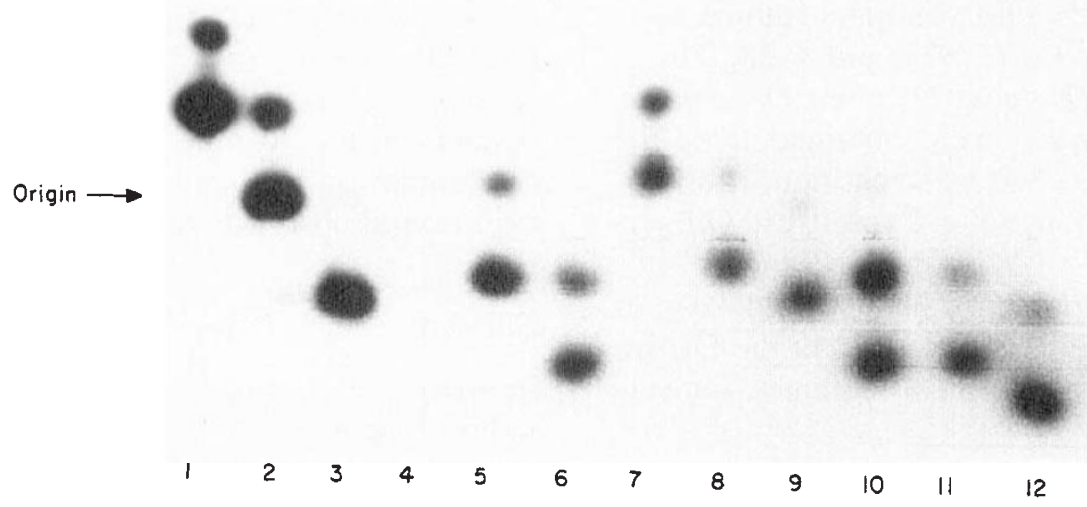

(a)

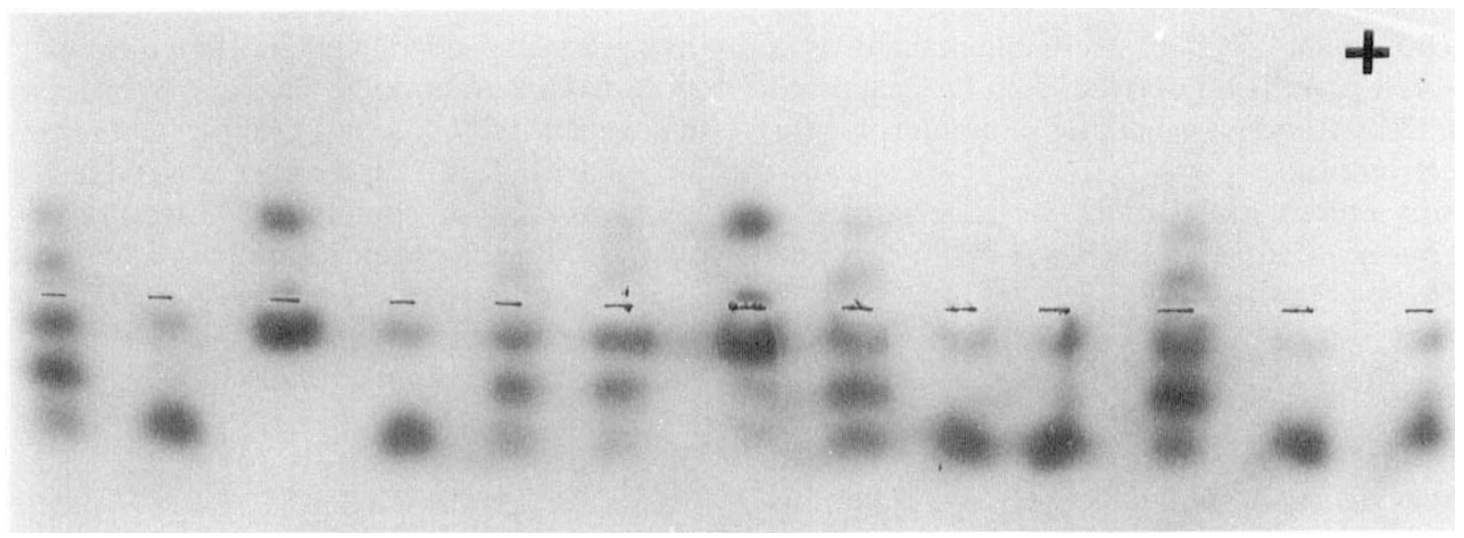

(b)

Fig. 1 Zymograms for alcohol dehydrogenase. (a) Eight species of the melanogaster species subgroup of Drosophila. From left, tracks 1-4: ADH-UF, ADH-F, ADH-S and ADH-null, respectively, of D. melanogaster. Tracks 5 and $6:$ ADH-F and ADH-S of D. mauritiana. The gel also shows the ADH of monomorphic species: track $7, D$. erecta; track $8, D$. orena; track $9, D$. teissieri; track $10, D$. simulans; track $11, D$. sechellia; track $12, D$. yakuba. (b) Allozymes of $D$. mauritiana. From left, the first three individuals are ADH-FS, ADH-S and ADH-F, respectively. (Positions of the insertion slots on the gel have been inked in.) 

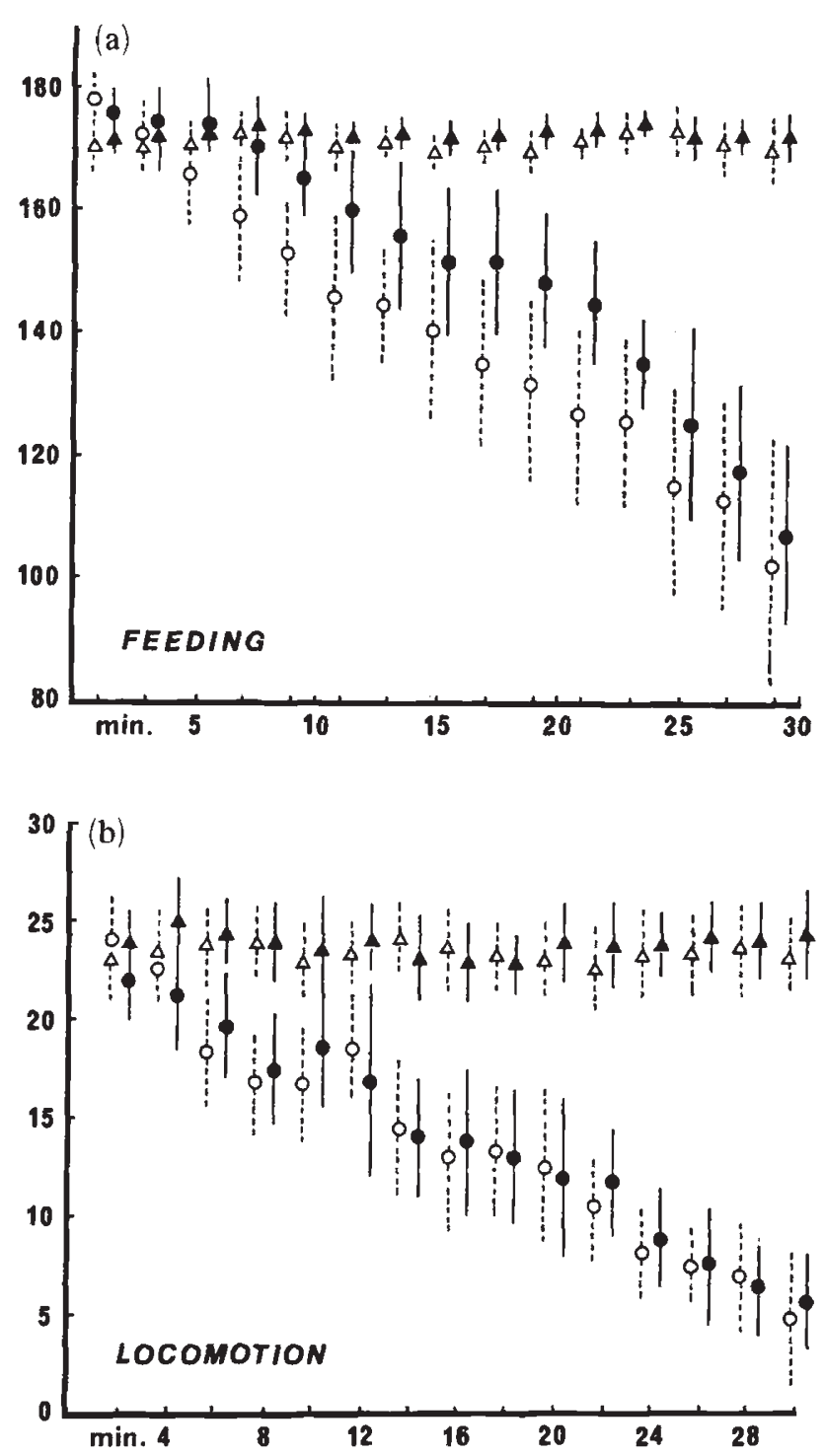

Fig. 2 Behaviour of early third instar larvae of Drosophila mauritiana: (a) Feeding activity, (b) locomotor activity. Triangles, yeast suspension. Circles, yeast suspension in 5 per cent ethanol. $A d h^{f}$ larvae, black symbols; $A d h^{\mathrm{S}}$ larvae, white symbols. Vertical bars delimit $\pm 2 \times \mathrm{SE}$.

Figure 2(a) shows that feeding rate was constant for both genotype groups over a $30 \mathrm{~min}$ observation period in live yeast suspension. After transfer to yeast suspension in 5 per cent ethanol, feeding rate declined in both strains. The results suggest that for $A d h^{\mathrm{F}}$ homozygotes the onset of the decline in feeding rate may be delayed until after about 5 minutes' exposure to ethanol whereas for the $A d h^{\mathrm{S}}$ homozygotes it appears to begin earlier. In fact the observational points for $A d h^{\mathrm{F}}$ larvae are consistently higher from about minute 7 to minute 29. There is a suggestion here that the feeding activity of $A d h^{\mathrm{F}}$ larvae may be less adversely affected by dietary ethanol than that of $A d h^{\mathrm{S}}$ larvae.

\section{Responses to ethanol by larvae of D. melanogaster and $\mathrm{D}$. simulans}

Larvae of the two cosmopolitan sibling species were measured during the same period of development as $D$. mauritiana. Both species were derived from wild populations of African origin: D. melanogaster (Sierra Leone) monomorphic for $\mathrm{ADH}-\mathrm{S}$, and $D$. simulans (Nairobi) monomorphic for the $\mathrm{ADH}$ ubiquitous in this species. Figures 3 and 4 show that for these species also the control rates of locomotor and feeding activity varied around a constant level in yeast suspension. Their responses to ethanol are different.

The locomotor activity of $D$. melanogaster larvae (Fig. 3b) was significantly higher during the first $20 \mathrm{~min}$ following transfer to the ethanol-yeast suspension. Although the mean locomotor activity scores are consistently above those for corresponding yeast control values in the following period (20-30 min), the variation between individual larvae increased. Locomotor activity of $D$. simulans larvae (Fig. $4 \mathrm{~b}$ ) rose significantly during the period of $0-15 \mathrm{~min}$ following transfer to ethanol-yeast suspension. This trend reversed during the ensuing period (15-30 $\mathrm{min})$, when the rates of locomotion fell to levels significantly below the yeast control.

The feeding rates of $D$. melanogaster larvae showed no directional trend during the first 20 min period after transfer to ethanol-yeast suspension. In the period from 20-30 min a tendency towards lower mean feeding rate was associated with increasing variation between individuals (Fig. 3a). The feeding activity of $D$. simulans larvae remained relatively constant during the first $10 \mathrm{~min}$ following transfer to ethanol-yeast (Fig. $4 \mathrm{a})$ and at a similar level to that in the yeast control. Over the ensuing period (10-30 min) there was a decrement in the mean feeding rate which fell from 150.3 retractions per min initially to 93.9 per min at minute 30 . This drop of 56.4 retractions per $\min$ in $D$. simulans larvae may be compared with falls of 76.9 and 70.7 retractions per $\min$ for the $D$. mauritiana larvae homozygous for $A d h^{\mathrm{S}}$ or $A d h^{\mathrm{F}}$, respectively, over the same period in ethanol-yeast (Fig. 2a).

\section{Larva to adult survival}

A comparison of tolerance to ethanol of strains of $D$. mauritiana homozygous for the two alternative alleles $A d h^{\mathrm{F}}$ and $A d h^{\mathrm{S}}$ was made by measuring larval survival to the adult stage. Figure 5 shows that low concentrations of dietary ethanol, up to 2-3 per cent, improved survival rate to the adult stage. This indicates that at low concentration, ethanol is used as a nutritional resource by $D$. mauritiana larvae. At concentrations above 2 per cent, dietary ethanol causes a reduction in 

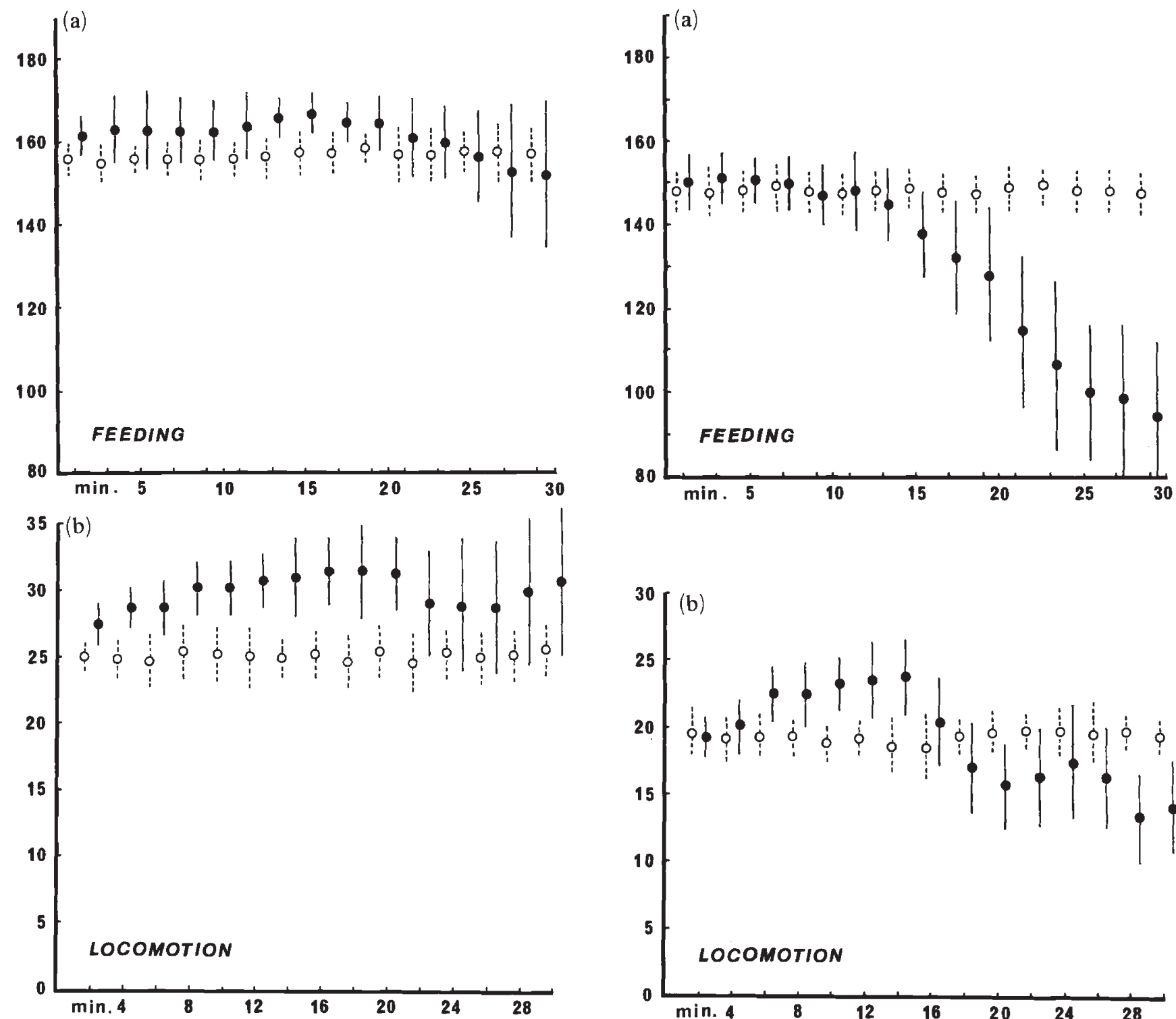

Fig. 3 Feeding (a) and locomotor activity (b) of early third instar larvae of Drosophila melanogaster in yeast (white circles), and yeast suspended in 5 per cent ethanol (black circles). Vertical bars delimit $\pm 2 \times \mathrm{SE}$.

the proportion of larvae surviving to the adult stage. No survivors were observed at concentrations exceeding 6 per cent ethanol. Pupal survival rates were virtually constant, so that survival to adult is very largely determined by larval survival up to pupariation. The response to ethanol of both genotype groups is complicated because the direction of the response is not the same over different ranges of ethanol concentration. Low concentrations of ethanol cause an increase, higher concentrations a decrease in survival rate. An analysis of variance using the angular transformation showed that: (i) there are significant differences in survival rate

between ethanol concentrations $\left(F_{4,90}=57, P<0.02\right)$; (ii) the two genotypes differ significantly in survival rate $\left(F_{1,90}=9.2, P<0.01\right)$; (iii) differences in ethanol concentration affect both genotypes in the same way $\left(F_{4,90}=0.1, \mathrm{NS}\right)$.

Inspection of Fig. 5 reveals that survival is considerably lower in larvae homozygous for $A d h^{\mathrm{S}}$ compared to those with $A d h^{\mathrm{F}}$ at all concentrations of ethanol including the zero control. Consequently much of the difference in survival between genotypes at higher ethanol concentrations results from this absolute difference in survival rate between them, rather than 
from a difference in response to ethanol itself. The question as to whether $A d h^{\mathrm{F}}$ confers higher alcohol tolerance relative to $A d h^{\mathrm{S}}$ was approached by expressing survival for each genotype at the higher concentra-

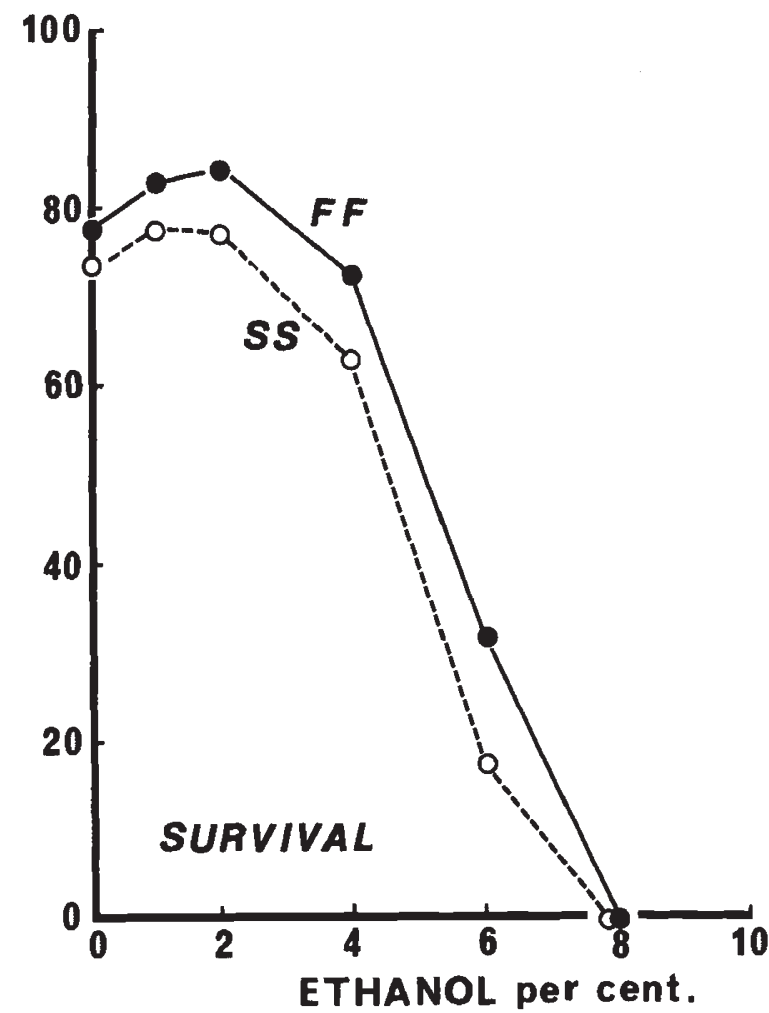

Fig. 5 Effects of ethanol on survival of larvae to the adult stage of Drosophila mauritiana. Survival rate is expressed as a percentage of the initial number of larvae at each concentration. Black symbols, $A d h^{\mathrm{S}}$ larvae; white symbols, $A d h^{\mathrm{F}}$ larvae.

Table 1 Analysis of variance for the effects of ethanol on survival of the $A d h^{\mathrm{S}}$ and $A d h^{\mathrm{F}}$ strains of Drosophila mauritiana

\begin{tabular}{lrrrr}
\hline & \multicolumn{3}{c}{ Mean survival (angles) } \\
\cline { 2 - 5 } Ethanol concentration & & \multicolumn{1}{c}{$A d h^{\mathrm{F}}$} & & $A d h^{\mathrm{s}}$ \\
\hline 4 per cent & & 66.8 & & 69.8 \\
6 per cent & & 30.7 & & 38.6 \\
Analysis of variance & & & & \\
\hline Source & d.f. & \multicolumn{1}{c}{$\mathrm{MS}$} & \multicolumn{1}{c}{$F$} & $P$ \\
\hline Ethanol concentration & 1 & 11352.2 & 48 & $<0.01$ \\
Genotype & 1 & 296.5 & 1.3 & $\mathrm{NS}$ \\
Interaction & 1 & 60.6 & 0.3 & $\mathrm{NS}$ \\
Error & 36 & 236.0 & & \\
\hline
\end{tabular}

Survival at each ethanol concentration was expressed as a proportion of the maximum (see Fig. 5). Values for each replicate were transformed to angles (degrees). tions as a proportion of the maximum reached at 2-3 per cent ethanol. Analysis of variance using the angular transformed data, shown in Table 1, confirms that an increase in ethanol concentration from 4 to 6 per cent significantly reduces survival, but that there is no significant difference between genotypes. There is, therefore, no evidence that the difference in molecular structure of alcohol dehydrogenase coded by the $A d h^{\mathrm{s}}$ and $A d h^{\mathrm{F}}$ alleles of $D$. mauritiana significantly affects ethanol-induced mortality.

\section{Discussion}

Thus far, $D$. simulans has proved to be monomorphic for $\mathrm{ADH}$ throughout its range, whereas $D$. melanogaster is regionally polymorphic. Other species of the melanogaster species subgroup endemic to the African continent ( $D$. teissieri, $D$. yakuba, D. erecta) though relatively widespread have not been so extensively studied and so it is possible that polymorphism may yet be detected. Existing stocks of $D$. orena in captivity are all derivatives of a single wild-caught female. Of the endemic island species, $D$. sechellia and $D$. mauritiana have proved to be monomorphic in several independent wild isolates. The variant allele $A d h^{\mathrm{F}}$ described here appears therefore to be the first reported example in a species of the subgroup, other than in D. melanogaster itself. Further examination of wild captures from Mauritius will be necessary to determine whether $\mathrm{ADH}$ polymorphism is present in the indigenous population of $D$. mauritiana, since the $A d h^{\mathrm{F}}$ allele could have arisen as a spontaneous mutation in the G93 stock since its isolation in the laboratory.

The alleles $A d h^{\mathrm{F}}$ and $A d h^{\mathrm{s}}$ of $D$. mauritiana specify alloenzymes of alcohol dehydrogenase which differ in their molecular structure. Information about the reaction kinetics of this enzyme in $D$. mauritiana given by Gelfand \& McDonald (1983), is presumably for the common $A d h^{\mathrm{S}}$ allele. It is not known whether the $A d h^{\mathrm{F}}$ allele differs significantly in its effect on ADH activity, but these alleles are indistinguishable in their effects on ethanol tolerance in D. mauritiana.

The evidence from breeding sites of wild populations is that $D$. melanogaster and $D$. simulans occupy different niches with respect to alcohol concentration (McKenzie \& Parsons, 1972; Parsons, 1977, 1981; Gibson \& Wilks, 1988). McKenzie \& McKechnie (1979) found only $D$. melanogaster larvae when ethanol concentration was about 7 per cent. When ethanol concentrations were less than 3 per cent, both $D$. melanogaster and $D$. simulans larvae were present. Drosophila simulans is less tolerant of ethanol than is D. melanogaster in terms of survival (David \& Bocquet, 1975, 1976). Drosophila melanogaster larvae are attracted to, and D. simulans larvae avoid, concentra- 
tions of ethanol around 6 per cent (Parsons, 1977, 1979; Parsons \& King, 1977; Green et al., 1983), and ethanol is less attractive to $D$. mauritiana at low concentrations than to $D$. simulans (Gelfand \& McDonald, 1983).

Dietary ethanol at the ecologically realistic concentration used here evokes changes in the behaviour of larvae of both $D$. melanogaster and $D$. simulans. Larvae respond on first contact with ethanol by an increase in locomotor activity, which may reflect aversion. In $D$. simulans larvae this is succeeded by a second phase of reduced locomotion, possibly caused by adverse physiological effects on their sensory, or motor, systems. Reduction in feeding rate may similarly be the consequence of aversion, or intoxication. Larvae of $D$. mauritiana exhibit behavioural responses to external environmental stimuli comparable with those of its cosmopolitan sibling species, but these responses are closer to those of $D$. simulans to which it is more nearly related. The two-phase response exhibited by $D$. simulans larvae was not observed in $D$. mauritiana larvae, which showed a consistent decline in locomotor activity beginning soon after ethanol was first ingested. A reduction in the feeding rate of simulans larvae began several minutes after contact with ethanol in the food medium, whereas there was no lag in the onset of the decline in feeding rates of mauritiana larvae.

Behavioural evidence complementary to this is that the transition from increasing to decreasing locomotor activity occurred earlier, and the reduction of locomotor activity was greater in $D$. simulans than in $D$. melanogaster. Moreover, depression of larval feeding rate began sooner in simulans and proceeded at a faster rate. The early onset and consistent decline in rates of locomotion and feeding shown by $D$. mauritiana larvae in ethanol also seems to be an indication of adverse physiological effects which later become manifest in their low survival rate. The survival rates of $D$. mauritiana larvae are broadly consistent with the evidence from larval behaviour and indicate that this species has a level of ethanol tolerance which is lower than that of the cosmopolitan sibling species $D$. melanogaster and D. simulans.

\section{Acknowledgements}

Thanks are due for support provided by the Ministry of Higher Education and Scientific Research of the Republic of Iraq to A.-Z.K. Muhammad-Ali.

\section{References}

ASHBURNER, M., BODMER, M. AND LEUMEUNIER, F. 1984. On the evolutionary relationships of Drosophila melanogaster. Devl. Genet., 4, 295-312.
DAVID, J. R. AND BOCQUET, C. 1975. Similarities and differences in latitudinal adaptation of two sibling Drosophila species. Nature, 257, 588-590.

DAVID, J. R. AND BoCQUET, C. 1976. Compound toxicities of different alcohols for two Drosophila sibling species: $D$. melanogaster and D. simulans. Comp. Biochem. Physiol., 54C, 71-74.

DAVID, J. R., De SCHEEMAEKER-LOUIS, M. AND PLA, E. 1980. Evolution in the seven species of the Drosophila melanogaster subgroup; comparison of the electrophoretic mobility of the enzymes produced by the $A d h$ locus. Drosoph. Inf. Serv., 55, 28-29.

DAVID, J. R., MERCOT, H., CAPY, P., McEVEY, S. F. AND HERREWEGE, J. VAN 1986. Alcohol tolerance and $A d h$ gene frequencies in European and African populations of Drosophila melanogaster. Génét. Sél. Évol., 18, 405-416.

GELFAND, L. J. AND McDONALD, J. F. 1983. Relationship between alcohol dehydrogenase $(\mathrm{ADH})$ activity and behavioural response to environmental ethanol in five Drosophila species. Behav. Genet., 13, 281-293.

GIBSON, J. B. AND WILKS, A. V. 1988. The alcohol dehydrogenase polymorphism of Drosophila melanogaster in relation to environmental ethanol tolerance and alcohol dehydrogenase activity. Heredity, 60, 403-414.

GREEN, C. H., BURNET, B. AND CONNOLLY, K. J. 1983. Organisation and patterns of inter- and intraspecific variation in the behaviour of Drosophila larvae. Anim. Behav., 31, 282-291.

HERREWEGE, J. VAN AND DAVID, J. R. 1985. Ethanol tolerance in Drosophila melanogaster: parallel variations in larvae and adults from natural populations. Drosoph. Inf. Serv., 61, 180-181.

LACHAISE, D., CARIOU, M.-L., DAVID, J. R., LEMEUNIER, F., TSACAS, L. AND ASHBURNER, M. 1988. Historical biogeography of the Drosophila melanogaster species subgroup. Evol. Biol., 22, 159-225.

McKENZIE, J. A. AND McKECHNIE, s. w. 1979. A comparative study of resource utilisation in natural populations of Drosophila melanogaster and D. simulans. Oecologia, 40 , 299-309.

McKeNZIE, J. A. AND PARSONS, P. A. 1972. Alcohol tolerance: an ecological parameter in the relative success of Drosophila melanogaster and D. simulans. Oecologia, 10, 373-388.

PARSONS, P. A. 1977. Larval reaction to alcohol as an indicator of resource utilisation differences between Drosophila melanogaster and D. simulans. Oecologia, 30, 141-146.

PARSONS, P. A. 1979. Larval reactions to possible resources in three species of Drosophila as indicators of ecological diversity. Aust. J. Zool., 27, 413-419.

PARSONS, P. A, 1981. Sympatric speciation in Drosophila? Ethanol threshold metrics and habitat subdivision. Am. Nat., 117, 1023-1026.

PARSONS, P. A. AND KING, S. B. 1977. Ethanol: larval discrimination between two Drosophila sibling species. Experientia, 33, 898-899.

SMITH, I. 1968. Chromatographic and Electrophoretic Techniques, vol. II, Zone Electrophoresis, 2nd edn. Heinemann Medical, Bath, UK. 